\title{
NOTIZEN
}

\section{Thermisches Verhalten von Alkalihexachlorotitanaten (IV)}

Von Paul Ehrlich und Eberhard Framm Institut für Anorganische Chemie der Techn. Hochschule Hannover

(Z. Naturforschg. 9 b, 326 [1954]; eingeg. am 26. März 1954)

Für die Herstellung von Titan interessiert es sehr, das Metall durch Elektrolyse von Chloridschmelzen gewinnen zu können. Voraussetzung dafür ist, daß geeignete, thermisch stabile Titandoppelchloride gefunden werden. Zunächst wurden von uns Darstellung und Eigenschaften der Alkalisalze mit vierwertigem Titan vom Typ $\mathrm{Alk}_{2}\left[\mathrm{TiCl}_{6}\right]$ untersucht und ihre Zersetzungsdrucke gemessen.

In Übereinstimmung mit der Literatur 1, 2 lassen sich allein die K-, Rb- und Cs-Salze durch Ausrühren aus mit $\mathrm{HCl}$ gesättigter Salzsäure (und zwar in wasserfreier Form) gewinnen. Bei der Synthese aus Alkalimetallchlorid und $\mathrm{TiCl}_{4}$ im Bombenrohr $\left(300^{\circ} \mathrm{C}\right)$ werden nur ganz geringe Umsetzungen erzielt; sie steigen vom K- über das Rb- zum Cs-Salz hin an $\left(0,5 \rightarrow 0,8 \rightarrow 0,9 \mathrm{Mol}_{0} \mathrm{TiCl}_{4}\right)$. Parallel damit geht die Hydrolysen-Beständigkeit der Salze; $\mathrm{Cs}_{9}\left[\mathrm{TiCl}_{6}\right]$ bleibt tagelang an Luft unverändert.

An den Präparaten wurden nach der statischen Methode mit einem Isoteniskop in einer Anordnung nach $\mathrm{F}$ is cher und Jübermann ${ }^{3}$ die Zersetzungsdrucke der Reaktion $\mathrm{Alk}_{2}\left[\mathrm{TiCl}_{6}\right] \rightarrow 2 \mathrm{Alk} \mathrm{Cl}+\mathrm{TiCl}_{4}$ gemessen. Sie gehorchen der Formel:

$$
\begin{aligned}
\mathrm{K}_{2}\left[\mathrm{TiCl}_{6}\right]: 10 \log \mathrm{p}_{(\text {Torr })} & =\frac{-6,02 \cdot 10^{3}}{\mathrm{~T}}+10,36, \\
\mathrm{Rb}_{2}\left[\mathrm{TiCl}_{6}\right]: 10 \log \mathrm{p}_{(\text {Torr })} & =\frac{-7,68 \cdot 10^{3}}{\mathrm{~T}}+11,53 .
\end{aligned}
$$

Daraus berechnen sich die Bildungswärmen von $27 \mathrm{kcal}$ für $\mathrm{K}_{2}\left[\mathrm{TiCl}_{6}\right]$ und $35 \mathrm{kcal}$ für $\mathrm{Rb}_{2}\left[\mathrm{TiCl}_{6}\right]$. Der Wert für $\mathrm{Cs}_{2}\left[\mathrm{TiCl}_{6}\right]$ wird auf $>40 \mathrm{kcal}$ abgeschätzt; doch konnten die Zersetzungsdrucke des Cs-Salzes, die bei entsprechend höheren Temperaturen $\left(600-700^{\circ} \mathrm{C}\right)$ liegen, experimentell nicht gemessen werden, da $\mathrm{Cs}_{2}\left[\mathrm{TiCl}_{6}\right]$ als Molekel

Die Deutsche Forschungsgemeins chaft unterstützte die Arbeit durch Bewilligung von Sachmitteln.

1 A. R os en he i m u. O. S c hü t t e, Z. anorg. Chem. 26, 242 [1901].

2 W. S e i d e l u. W. F is c h e r, Z. anorg. allg. Chem. 247, 367 [1941],

3 W. F is cher (Hannover) u. O. Jü be r m ann (Leverkusen), Privatmitteilung. schon eine zu starke Flüchtigkeit zeigt. Das Salz schmilzt bei $\sim 570^{\circ} \mathrm{C}$ zu einer klaren, braunen Schmelze; der Schmelzpunkt des $\mathrm{Rb}_{2}\left[\mathrm{TiCl}_{6}\right]$ liegt höher, bei $\sim 605^{\circ} \mathrm{C}$.

\section{Zur Frage der Äquator-Kleinwinkelinterferenz an Polyvinylchlorid und Polyacrylnitril}

Von O. Kratky, A. S ekora, E. Treiber und W. B e r n d t

Institut für theoretische und physikalische Chemie der Universität Graz

(Z. Naturforschg. 9 b, 326 [1954]; eingeg. am 14. April 1954)

Durch eine Reihe von Beobachtungen ( $\mathrm{H}$ e s s, K i e s sig, Z a hn, Fankuchen, M ark, Arnett, Meibohm u. a.) konnte die Tatsache bestätigt werden, daß fast alle vollsynthetischen Fasern meridiale Interferenzen großer Periode geben 1 .

In einer kurzen vorausgegangenen Notiz 2 haben wir über Äquatorinterferenzen im Kleinwinkelgebiet an Polyacrylonitril, Polyvinylchlorid und einem Mischpolymerisat beider berichtet, deren Deutung offen gelassen wurde. Ähnliche Effekte sind bisher lediglich an Seide gefunden worden, die dort eindeutig von sehr geringen Mengen Seidenwachs hervorgerufen werden und nach längerer Extraktion mit Äther-Alkohol verschwinden 3 .

Im Bestreben, den mitgeteilten Effekt aufzuklären, haben wir die Fasern - durchweg technische Produkte sehr sorgfältigen Extraktionen in Äther, Alkohol, Tetrachlorkohlenstoff und warmem Seifenwasser unterzogen und konnten feststellen, daß auch hier die Interferenzen zum Verschwinden gebracht werden können. (Im Falle des Polyvinylchlorids erfährt dabei allerdings auch das Weitwinkeldiagramm schwache Veränderungen.)

Wenn auch eine endgültige Deutung der äquatorialen Kleinwinkelinterferenzen noch nicht gegeben werden kann, so sei doch auf die Möglichkeit hingewiesen, daß es sich um Effekte von orientiert eingelagerten Weichmachern, Gleit- und Ausrüstungsmittel handelt, die der Faser von der Fabrikation bzw. Ausrüstung her anhaften. Da die meisten dieser Stoffe Derivate langkettiger Paraffincarbonsäuren u. ä. sind, wäre das Auftreten von Kleinwinkelinterferenzen denkbar.

1 Vgl. K. Hess u. H. Ki ess ig, Kolloid-Z. 130 , 10 [1953].

2 O. Kratky, A. S ekora u. E. Treiber, Z. Naturforschg. 8 b, 615 [1953].

3 O. K r a tky, E.S chauenstein u. A. Sekora Nature [London] 170, 796 [1952]. 